\title{
MicroRNA-876 is sponged by long noncoding RNA LINC00707 and directly targets metadherin to inhibit breast cancer malignancy
}

This article was published in the following Dove Press journal: Cancer Management and Research

\author{
Tong $\mathrm{Li}^{\mathrm{I}}$ \\ Yunpeng $\mathrm{Li}^{2}$ \\ Hongyan Sun' \\ 'Department of General Surgery, The \\ Fourth People's Hospital of Jinan, Jinan, \\ Shandong 25003I, People's Republic of \\ China; ${ }^{2}$ Department of General Surgery, \\ Ningjin County People's Hospital, Ningjin, \\ Shandong 253400, People's Republic of \\ China
}

Background: MicroRNA-876-5p (miR-876) dysregulation contributes to the aggressiveness of various types of human cancer. This study was aimed at measuring miR-876 expression in breast cancer, determining the specific roles of miR-876 in the progression of breast cancer and understanding the corresponding molecular mechanisms.

Materials and methods: miR-876 expression in breast cancer tissues and cell lines was quantified via RT-qPCR. The effect of miR-876 upregulation on the malignant phenotype of breast cancer cells was investigated using CCK-8 assays, flow cytometry, Transwell migration and invasion assays and tumor xenograft experiments. The mechanisms underlying the tumor-suppressive action of miR-876 in breast cancer cells were explored using bioinformatic analysis, luciferase reporter assays, RT-qPCR and Western blot analysis. Results: miR-876 was found to be underexpressed in breast cancer tissues and cell lines. Decreased miR-876 expression notably correlated with lymphatic invasion metastasis, TNM stage and differentiation grade. Overall survival was lower among patients with breast cancer and low miR-876 expression than in patients with high miR-876 expression. Restoration of miR-876 expression decreased breast cancer cell proliferation, migration and invasion in vitro and restricted tumor growth in vivo as well as increased cell apoptosis. Metadherin (MTDH) was identified as a novel target of miR-876 in breast cancer cells. Furthermore, long intergenic nonprotein-coding RNA 707 (LINC00707) acted as a molecular sponge for miR-876, thereby regulating MTDH expression in breast cancer. Finally, silencing miR-876 expression attenuated the influence of a LINC00707 knockdown on the malignancy of breast cancer cells

Conclusion: This study, thus, revealed the vital functions of the LINC00707-miR-876-MTDH pathway in breast cancer and provided attractive targets and markers for its treatment.

Keywords: breast cancer, microRNA-876-5p, metadherin, long intergenic non-proteincoding RNA 707, therapeutic target

\section{Introduction}

Breast cancer, which is derived from mammary epithelial tissue, is one of the most prevalent human malignant tumors among women worldwide. ${ }^{1}$ Morbidity and mortality among patients with breast cancer in China accounts for approximately $12.2 \%$ and $9.6 \%$ of global figures, respectively. ${ }^{2-4}$ In the past few decades, considerable advances in the diagnosis and treatment have led to an obvious improvement in the clinical outcomes of patients with breast cancer. Unfortunately, to date, breast cancer is still a refractory disease, mainly owing to its recurrence, metastasis and tolerance to radiotherapy and
Department of General Surgery, The

Fourth People's Hospital of Jinan, 52

Shifan Road, Jinan, Shandong 25003I,

People's Republic of China

Tel +86I 509877 I66I

Emailsunhy_810612@I63.com 
chemotherapy. ${ }^{5-7}$ Multiple factors, including the activation of oncogenes, deactivation of tumor suppressors and other hereditary and epigenetic alterations, are involved in the initiation and progression of breast cancer. ${ }^{8-10}$ However, details of the underlying mechanisms regulating breast cancer progression are not yet fully understood. Thus, a comprehensive understanding of the molecular mechanisms underlying breast cancer pathogenesis is crucial for the identification of novel targets for precision therapy.

MicroRNAs (miRNAs) are a group of evolutionarily conserved, noncoding and short RNA molecules 19-24 nucleotides long. ${ }^{11}$ miRNAs act as gene expression regulators by imperfect or near-perfect base pairing with the 3 '-UTRs of their target mRNAs, thereby resulting in translation suppression and/or mRNA degradation. ${ }^{12}$ Multiple lines of evidence have demonstrated that nearly all cellular physiological and pathological processes are closely regulated by miRNAs including carcinogenesis and cancer progression. ${ }^{13-15}$ The aberrant expression of miRNAs is a common and important feature of breast cancer, and their dysregulation participates in the malignant phenotypes of breast cancer by acting as oncogenes or tumor suppressors. ${ }^{16-18}$ Therefore, silencing of oncogenic miRNAs and restoring the expression of tumorsuppressing miRNAs might be a promising therapeutic method for patients with breast cancer.

Long noncoding RNAs (lncRNAs) are another group of noncoding RNA transcripts that are implicated in the regulation of various pathological processes, by performing important functions in the malignant progression of human cancers. ${ }^{19}$ It is well-known that lncRNAs are able to serve as competing endogenous RNAs (ceRNAs) to modulate the expression of genes by sponging miRNAs, thus titrating available miRNAs and contributing to tumorigenesis, including breast carcinogenesis. ${ }^{20-22}$ Accordingly, further exploration of the specific functions of lncRNAs in breast cancer is likely to provide effective targets for the development of therapies.

Abnormal expression of miR-876-5p (miR-876) contributes to the aggressiveness of multiple types of human cancer, including osteosarcoma, ${ }^{23}$ hepatocellular carcinoma, ${ }^{24}$ lung cancer, $^{25}$ and head and neck squamous cell carcinoma. $^{26}$ However, whether miR-876 is aberrantly expressed in breast cancer and its mechanisms of action have not yet been investigated. In this article, we provided experimental evidence that miR-876 is sponged by LINC00707 and can restrain breast cancer progression through metadherin (MTDH) downregulation.

\section{Materials and methods}

\section{Ethics statement}

The Ethics Committee of The Fourth People's Hospital of Jinan approved the study protocol, and written informed consent was provided by all individual participants prior to surgical resection. All the experimental procedures were in accordance with the Declaration of Helsinki.

\section{Tissues and cell lines}

In total, 53 pairs of breast cancer samples and adjacent normal tissues were collected from breast cancer patients at The Fourth People's Hospital of Jinan who had not been treated with any preoperative therapies, such as radiotherapy or chemotherapy. The tissue samples were snap-frozen in liquid nitrogen after surgical excision and stored at $-80^{\circ} \mathrm{C}$ until total RNA and protein isolation.

The four breast cancer cell lines, MCF-7, MDA-MB-231, BT-474 and SKBR3, and human breast epithelial cell line MCF-10A were purchased from the Chinese Academy of Sciences (Shanghai, China). All the cell lines were kept at $37^{\circ} \mathrm{C}$ in a humidified chamber with $5 \% \mathrm{CO}_{2}$ and were grown in DMEM containing $10 \% \mathrm{FBS}$ and $1 \%$ penicillin-streptomycin mixture (all from Gibco; Thermo Fisher Scientific, Inc., Waltham, MA, USA).

\section{Transfection}

The miR-876 agomir (agomir-876), negative control agomir (agomir-NC), miR-876 antagomir (antagomir-876) and antagomir-NC were acquired from Shanghai GenePharma Co., Ltd. (Shanghai, China). The small interfering RNA (siRNA) targeting LINC00707 (si-LINC00707) and the negative control siRNA (si-NC) were chemically synthesized by Guangzhou RiboBio Co. Ltd. (Guangzhou, China). The MTDH sequence lacking its 3'-UTR was produced by Guangzhou RiboBio Co. Ltd., inserted into the pcDNA3.1 plasmid, and the resulting plasmid was named pcDNA3.1-MTDH (pc-MTDH). Cells were seeded in 6-well plates $24 \mathrm{hrs}$ prior to transfection. Transient transfection was performed using Lipofectamine ${ }^{\circledR} 2000$ (Thermo Fisher Scientific, Inc.), as per the manufacturer's protocol.

\section{RNA isolation and RT-qPCR}

Total RNA was isolated with TRIzol ${ }^{\circledR}$ Reagent (Thermo Fisher Scientific, Inc.), and the concentration and quality were determined using a Nanodrop ${ }^{\circledR}$ ND-1000 spectrophotometer (NanoDrop Technologies; Thermo Fisher Scientific, Inc). miR-876 expression was determined by 
means of the Mir-X ${ }^{\mathrm{TM}}$ miRNA qRT-PCR TB Green ${ }^{\mathrm{TM}}$ Kit (Takara Biotechnology Co., Ltd., Dalian, China) with small nuclear RNA U6 as the control. MTDH mRNA and LINC00707 expression were quantified by reverse transcription and qPCR with the PrimeScript ${ }^{\circledR}$ RT reagent kit (Takara Biotechnology Co.) and $\mathrm{SYBR}^{\circledR}$ Premix Ex Taq $^{\text {TM }}$ II Kit (Takara Biotechnology Co.), respectively. Relative MTDH mRNA and LINC00707 expression levels were normalized to GAPDH. The $2^{-\Delta \Delta \mathrm{Cq}}$ method was used to analyze relative gene expression. ${ }^{27}$

\section{Cell counting kit-8 (CCK-8) assay}

Transfected cells were collected and separately seeded in 96-well microtiter plates (Corning Incorporated, Corning, NY, USA) at a density of $3 \times 10^{3}$ cells per well. The CCK- 8 assay was performed to evaluate cell proliferation at 4 consecutive time points: $0,24,48$ and $72 \mathrm{hrs}$ after seeding. Briefly, $10 \mu \mathrm{L}$ of the CCK-8 reagent (Dojindo, Gaithersburg, MD) was added into each well and the cells were incubated at $37^{\circ} \mathrm{C}$ and $5 \% \mathrm{CO}_{2}$ for another 2 hrs. Absorption at a $450 \mathrm{~nm}$ wavelength was measured on a microplate reader (Bio-Rad, Hercules, CA, USA).

\section{Flow cytometric analysis of apoptosis}

After $48 \mathrm{hrs}$ culture, the transfected cells were harvested, washed three times with ice-cold PBS and analyzed for cell apoptosis using the Annexin V-FITC Apoptosis Detection Kit (BioLegend, San Diego, CA, USA). In particular, the transfected cells were resuspended in $100 \mu \mathrm{L}$ binding buffer supplemented with $5 \mu \mathrm{L}$ Annexin V-FITC and $5 \mu \mathrm{L}$ propidium iodide. Following 30-min incubation at room temperature in the dark, the prevalence of apoptosis was determined using a flow cytometer (FACScan, BD Biosciences, Heidelberg, Germany). CellQuest software (BD Biosciences, Heidelberg, Germany) was employed to analyze the data.

\section{Transwell migration and invasion assays}

Migration and invasion were examined using a 24-well Transwell chamber (Corning Costar, Corning, NY, USA) containing a polycarbonate membrane filter (pore size: $8 \mu \mathrm{m}$ ). Matrigel (BD Biosciences, Franklin Lakes, NJ, USA)-coated chambers were used for the invasion assay, while the chambers in the migration assay were not coated with Matrigel. For both assays, a total of $5 \times 10^{4}$ transfected cells were seeded in the upper chambers. The bottom chambers were filled with $500 \mu \mathrm{L}$ DMEM containing 10\% FBS. The cells were incubated at $37^{\circ} \mathrm{C}$ and $5 \% \mathrm{CO}_{2}$ for $24 \mathrm{hrs}$. The nonmigratory and noninvading cells were gently removed with a cotton swab, whereas the migratory and invading cells were fixed in $70 \%$ ethanol, stained with $0.1 \%$ crystal violet and photographed under an inverted light microscope (Olympus Corporation, Tokyo, Japan). Finally, the migratory or invading cells in five randomly selected visual fields were counted.

\section{Tumor xenograft experiments}

The procedures for all animal experiments were approved by the Animal Research Committee of The Fourth People's Hospital of Jinan and were conducted in accordance with the Animal Protection Law of the People's Republic of China-2009 for experimental animals. MCF-7 cells transfected with agomir-876 or agomir-NC were harvested 24 hrs post-transfection and implanted into the dorsal flank of nude mice that were purchased from Vital River Laboratory Animal Technology (Beijing, China). The agomir-876 group was injected with miR-876-overexpressing cells, whereas the agomir-NC group was injected with agomir-NC-expressing cells. Four weeks following the cell injection, all the nude mice were euthanized under anesthesia, and the tumor weights and volumes were measured. Tumor volume was calculated using the following formula: tumor volume $=\left(\right.$ width $^{2} \times$ length $) / 2$.

\section{Bioinformatic analysis}

Three miRNA target prediction bioinformatics tools, TargetScan (http://targetscan.org/), microRNA. org (http://www.microrna.org/microrna/microrna/home. do), and StarBase 3.0 (http://starbase.sysu.edu.cn/), were used to search for the putative target of miR-876.

StarBase 3.0 (http://starbase.sysu.edu.cn/) and Lnc Base Experimental v.2 (http://carolina.imis.athenainnovation.gr/diana_tools/web/index.php? $\mathrm{r}=\operatorname{lncb}$ asev $2 \%$ 2findex-experimental) were employed to investigate the miR-876-LINC00707 axis.

\section{A luciferase reporter assay}

The 3'-UTR fragments of $M T D H$ containing the wild-type (WT) or mutant (MUT) miR-876-binding site were amplified by Shanghai GenePharma Co. Ltd. and cloned into the psiCHECK2 vector (Promega Corporation, Madison, WI, USA). These luciferase reporter plasmids were designated as MTDH-WT and MTDH-MUT, respectively. LINC00707WT and LINC00707-MUT reporter plasmids were also produced by Shanghai GenePharma Co. Ltd. Cells were seeded in 24-well plates and co-transfected with the luciferase reporter plasmids and agomir-876 or agomir-NC by means of Lipofectamine ${ }^{\circledR} 2000$. Finally, the cells were collected, and 
luciferase activity was detected using the Dual Luciferase Reporter Assay (Promega Corporation, Madison, WI, USA). Firefly luciferase activity was normalized to Renilla luciferase activity.

\section{Western blotting}

Cell lysates were prepared using radioimmunoprecipitation assay lysis buffer (Beyotime Institute of Biotechnology, Shanghai, China). The total protein concentration was measured using the BCA Protein Assay kit (Pierce; Thermo Fisher Scientific, Inc.). Equivalent amounts of protein were resolved by SDS-PAGEin a $10 \%$ gel, transferred onto polyvinylidene difluoride membranes and blocked at room temperature with a $5 \%$ nonfat milk solution. After incubation overnight at $4{ }^{\circ} \mathrm{C}$ with primary antibodies against MTDH (cat. \# ab227981; Abcam; Cambridge, MA, USA) or GAPDH (cat. \# ab128915; Abcam), the membranes were probed with a goat anti-rabbit IgG horseradish peroxidaseconjugated secondary antibody (cat. \# ab6721; Abcam) at room temperature for $1 \mathrm{hr}$. Finally, an enhanced chemiluminescence detection system (ECL; Bio-Rad Laboratories, Inc.) was employed to visualize the protein signals.

\section{Statistical analysis}

Each experiment was repeated at least three times. All data are expressed as mean \pm SD and were analyzed in the SPSS software, version 16 (SPSS, Inc., Chicago, IL, USA). Pearson's $\chi^{2}$ test was conducted to determine the association between miR876 and clinical characteristics among patients with breast cancer. Correlation between miR-876 and MTDH as well as LINC00707 expression in the same breast cancer tissues was examined via Spearman's correlation analysis. The KaplanMeier method along with the log-rank test was applied to assess the prognostic value of miR-876 in patients with breast cancer. $P<0.05$ was assumed to indicate a statistically significant difference.

\section{Results}

\section{Expression profile of miR-876 in breast cancer and its associations with clinical factors}

To obtain an overview of the expression profile of miR-876, we first measured its expression in 53 pairs of breast cancer samples and adjacent normal tissues. An obvious decrease in miR-876 expression was identified in breast cancer tissue samples compared with that in adjacent normal tissue samples (Figure 1A, $P<0.05$ ). Similarly, the expression of miR-876 was significantly lower in MCF-7, MDA-MB-231, BT-474 and SKBR3 cell lines than in human breast epithelial cell line MCF-10A, especially in the MCF-7 and MDA-MB-231 cell lines (Figure 1B, $P<0.05$ ). Therefore, the two cell lines were chosen for further experiments.

We next investigated whether miR-876 expression in breast cancer tissues correlated with clinical factors. All breast cancer patients were classified into either low- or high-miR876 expression groups, based on the median miR-876 expression level in breast cancer tissues. There was no significant correlation between miR-876 expression and age $(P=0.158)$, or
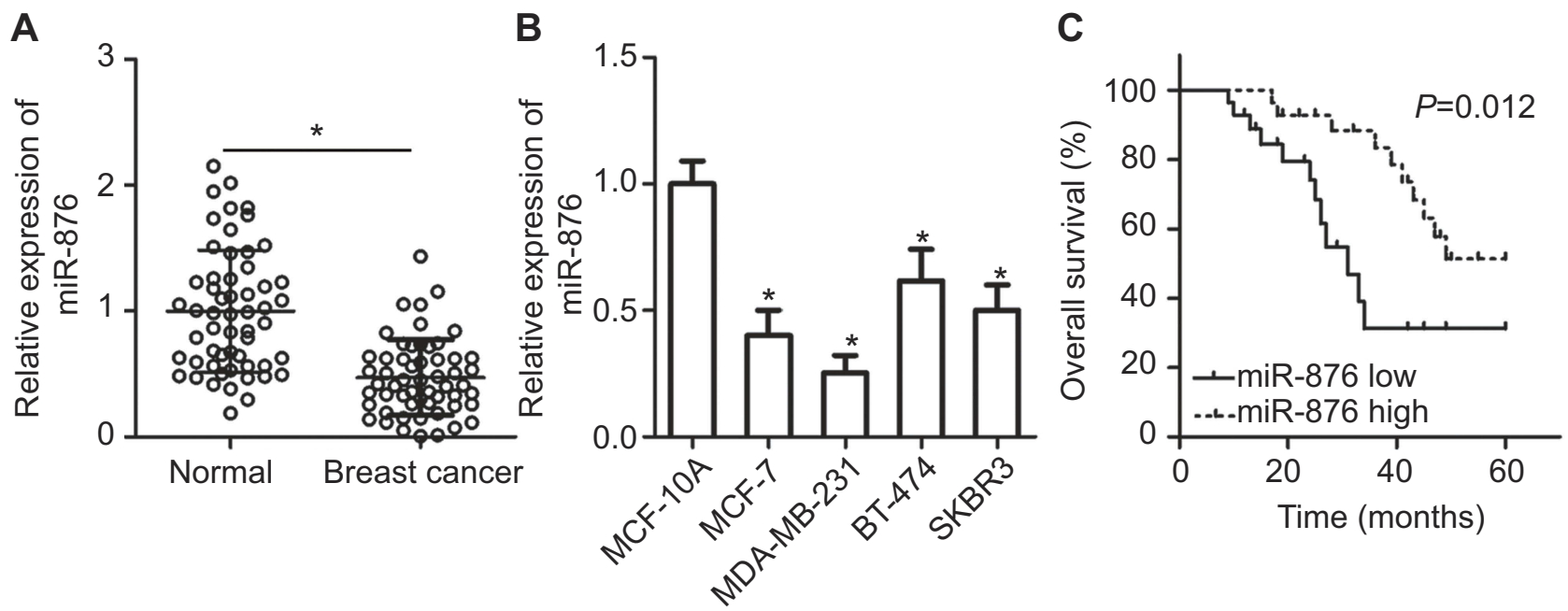

Figure I miR-876 is underexpressed in breast cancer tissues and cell lines. (A) RT-qPCR showing the expression profile of miR-876 in 53 pairs of breast cancer tissue samples and adjacent normal tissues. $* P<0.05$ as compared with normal tissues. (B) Expression levels of miR-876 in breast cancer cell lines, including MCF-7, MDA-MB-23I, BT-474 and SKBR3, as measured by RT-qPCR. Human breast epithelial cell line MCF-IOA served as the control. $* P<0.05$ as compared with MCF-I0A. (C) The overall survival of breast cancer patients in the low- or high-miR-876 expression groups. $P=0.012$.

Abbreviation: RT-qPCR, reverse transcription-quantitative polymerase chain reaction. 
tumor size $(P=0.412)$ of patients with breast cancer. However, a significant correlation was observed between decreased miR876 expression and lymphatic invasion metastasis $(P=0.002)$, TNM stage $(P=0.034)$ and differentiation grade $(P=0.029$; Table 1). We also analyzed the overall survival rate of breast cancer patients with low or high miR-876 expression levels. The results revealed that patients with breast cancer and low miR-876 expression had shorter overall survival than patients with relatively high levels of miR-876 expression (Figure 1C, $P=0.012$ ). These observations suggested that aberrant expression of miR-876 may be closely related to the initiation and progression of breast cancer.

\section{miR-876 upregulation inhibits breast} cancer cell growth and metastasis in vitro

To manipulate the expression of miR-876 in breast cancer cells, synthetic agomir-876 was introduced into MCF-7 and MDA-MB-231 cells. RT-qPCR analysis clearly confirmed that miR-876 was effectively upregulated in MCF-7 and MDAMB-231 cells after agomir-876 transfection (Figure 2A, $P<0.05)$. A CCK-8 assay for cellular proliferation showed that ectopic miR-876 expression notably decreased the proliferation of MCF-7 and MDA-MB-231 cells (Figure 2B, $P<0.05)$. The influence of miR-876 overexpression on breast cancer cell apoptosis was next examined via flow cytometric

Table I The association between miR-876 and clinical characteristics among patients with breast cancer

\begin{tabular}{|l|l|l|l|}
\hline \multirow{2}{*}{ Characteristics } & \multicolumn{2}{|l|}{ miR-876 expression } & \multirow{2}{*}{ P-value } \\
\cline { 2 - 3 } & Low & High & \\
\hline $\begin{array}{l}\text { Age (years) } \\
<50 \\
\geq 50\end{array}$ & 7 & 12 & 0.158 \\
\hline $\begin{array}{l}\text { Tumor size (cm) } \\
<2\end{array}$ & 20 & 14 & \\
$\geq 2$ & 10 & 13 & 0.412 \\
\hline $\begin{array}{l}\text { Lymphatic invasion metastasis } \\
\text { Negative }\end{array}$ & 17 & 13 & \\
Positive & 10 & 21 & $0.002^{\mathrm{a}}$ \\
\hline $\begin{array}{l}\text { TNM stage } \\
\text { I-II } \\
\text { III }\end{array}$ & 17 & 5 & \\
\hline $\begin{array}{l}\text { Differentiation grade } \\
\text { Well/moderately } \\
\text { Poorly/undifferentiated }\end{array}$ & 12 & 19 & $0.034^{\mathrm{a}}$ \\
\hline
\end{tabular}

Note: ${ }^{a} p<0.05$. analysis. As presented in Figure 2C, the proportion of apoptotic cells increased when miR-876 was overexpressed in MCF-7 and MDA-MB-231 cells $(P<0.05)$. Furthermore, Transwell migration and invasion assays indicated that agomir-876 transfection led to obvious reductions in MCF-7 and MDA-MB -231 cell migration and invasion (Figure $2 \mathrm{D}$ and $\mathrm{E}, P<0.05$ ). These results collectively meant that miR-876 functions as a tumor-suppressing miRNA in breast cancer.

\section{MTDH is a direct target of miR-876 in breast cancer cells}

As for the mechanism, miRNAs are known to regulate the expression of their targets. ${ }^{12}$ Accordingly, identifying the direct target gene of miR-876 in breast cancer cells was essential for understanding its role in the aggressiveness of breast cancer. Bioinformatics analysis predicted that the 3'-UTR of $M T D H$ mRNA contains a complementary binding site for miR-876 (Figure 3A). The luciferase reporter assay was conducted to determine whether $M T D H$ mRNA was a direct target of miR-876 in breast cancer. The results revealed that transfection of agomir-876 significantly reduced the luciferase activity of MTDH-WT in MCF-7 and MDA-MB-231 cells $(P<0.05)$, whereas cotransfection of agomir-876 and MTDH-MUT did not affect luciferase activity (Figure $3 \mathrm{~B}$ and $\mathrm{C}$ ).

We next evaluated the potential association between miR876 and $M T D H$ in breast cancer. RT-qPCR analysis indicated that $M T D H$ mRNA expression was higher in breast cancer tissue samples than in adjacent normal tissues (Figure 3D, $P<0.05$ ). Notably, an inverse correlation was observed between miR-876 and MTDH mRNA in breast cancer tissue samples, as demonstrated by Spearman's correlation analysis (Figure 3E; $\mathrm{R}^{2}=0.3348, P<0.0001$ ). Furthermore, compared with cells transfected with agomir-NC, MTDH expression was suppressed at both mRNA (Figure 3F, $P<0.05$ ) and protein levels (Figure 3G, $P<0.05$ ) in MCF-7 and MDA-MB-231 cells when transfected with agomir-876. These results meant that $M T D H$ mRNA is a direct target of miR-876 in breast cancer.

\section{Restoring MTDH expression reduces miR-876-mediated inhibition of breast cancer cell growth and metastasis in vitro} To test whether MTDH is involved in miR-876's tumorsuppressive effects in breast cancer, we restored MTDH expression in miR-876-overexpressing MCF-7 and MDAMB-231 cells by cotransfection with the MTDH overexpression plasmid pc-MTDH. Unsurprisingly, upregulation of miR876 noticeably decreased MTDH protein expression in MCF-7 

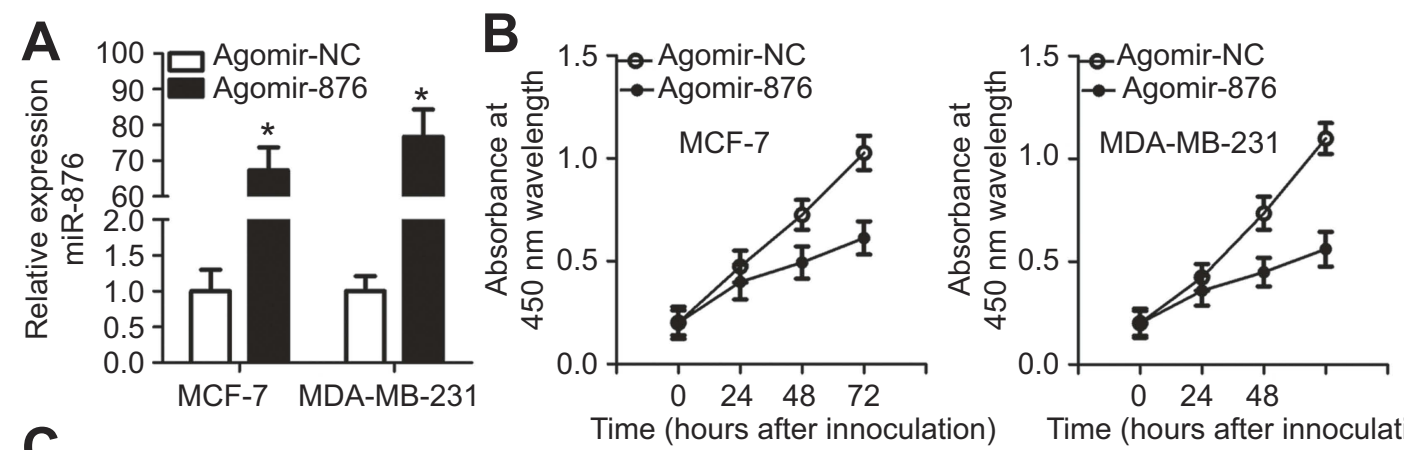

C
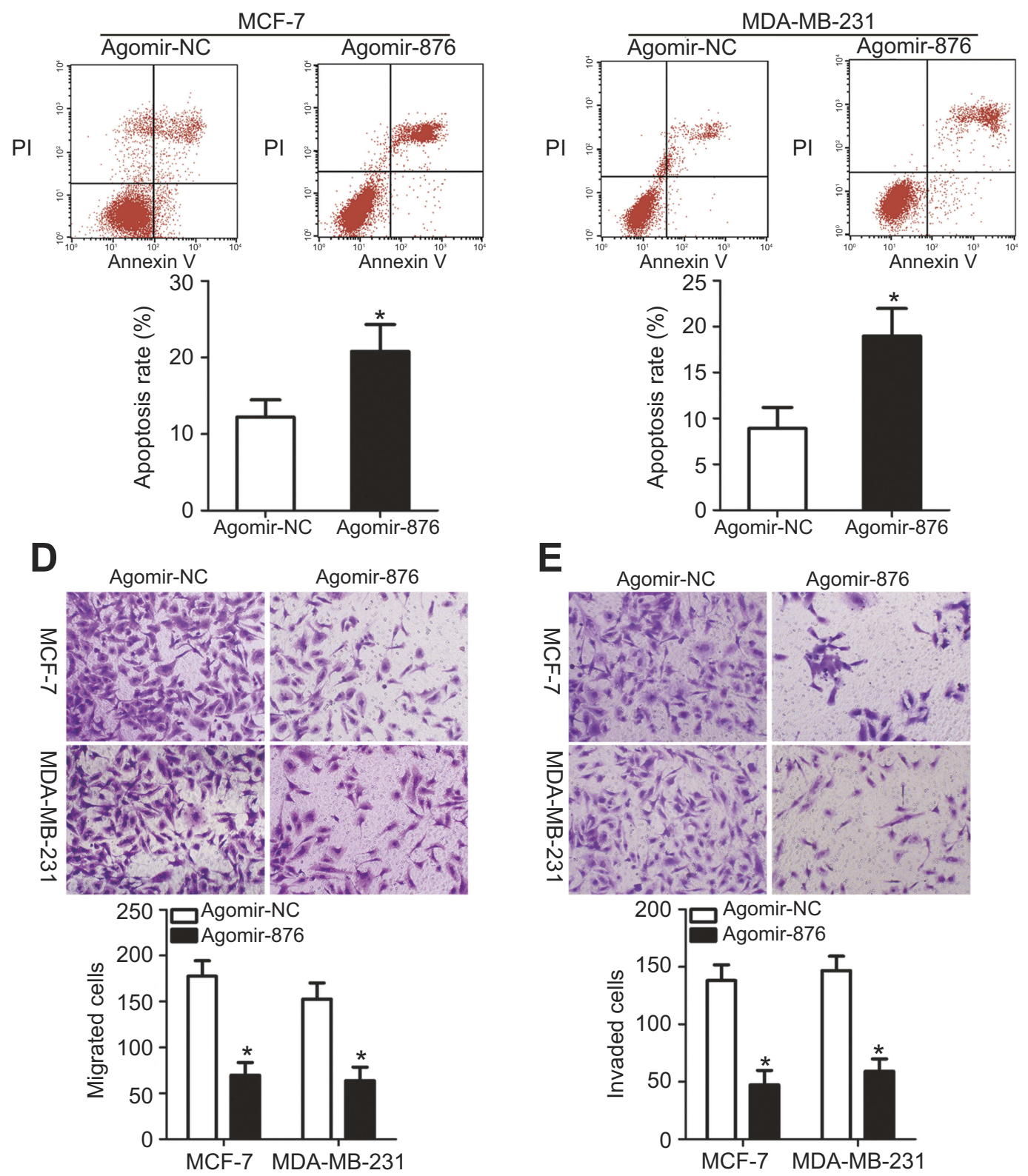

Figure 2 miR-876 overexpression decreases breast cancer cell proliferation, migration and invasion and increases apoptosis in vitro. (A) MCF-7 and MDA-MB-23I cells were treated with agomir-876 or agomir-NC. Their total RNA was isolated after 48 hrs of incubation, and transfection efficiency was determined by RT-qPCR. $* P<0.05$ in comparison with agomir-NC. (B, C) The proliferation and apoptosis of miR-876-overexpressing MCF-7 and MDA-MB-23I cells were determined by CCK-8 assays and flow cytometry. ${ }^{*} P<0.05$ as compared with agomir-NC. (D, E) Migration and invasion abilities were examined in MCF-7 and MDA-MB-23I cells after transfection with agomir-876 or agomir-NC. $* P<0.05$ relative to group agomir-NC.

Abbreviations: agomir-876, miR-876 agomir; agomir-NC, negative control agomir; RT-qPCR, reverse transcription-quantitative polymerase chain reaction. 
A

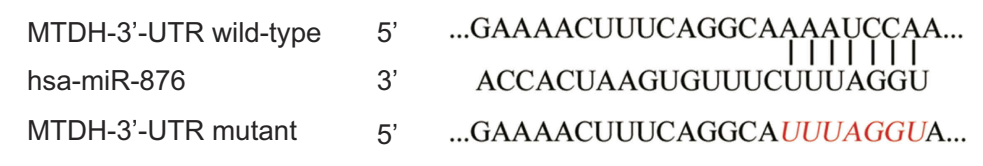

C

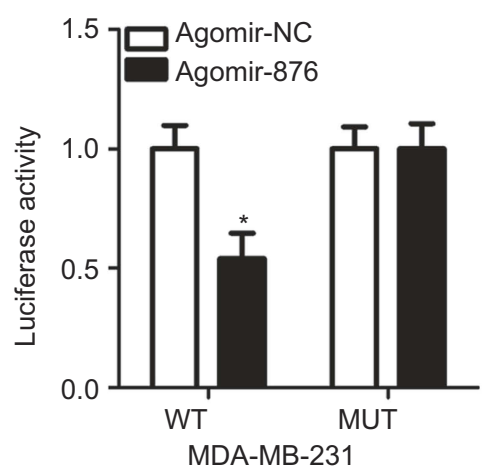

F

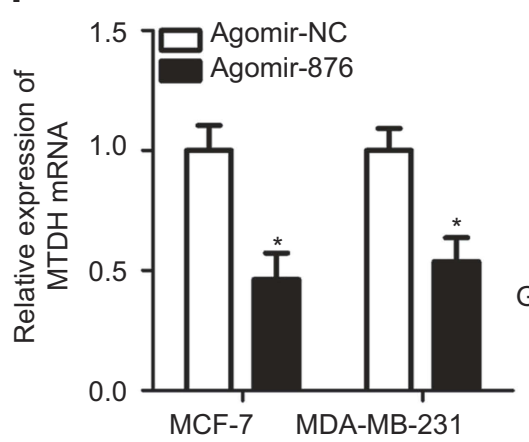

B MCF-7

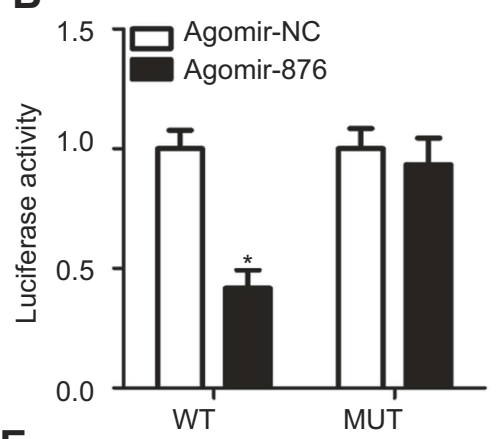

E

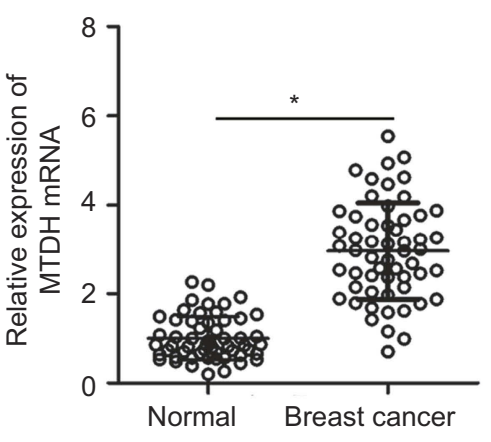

G
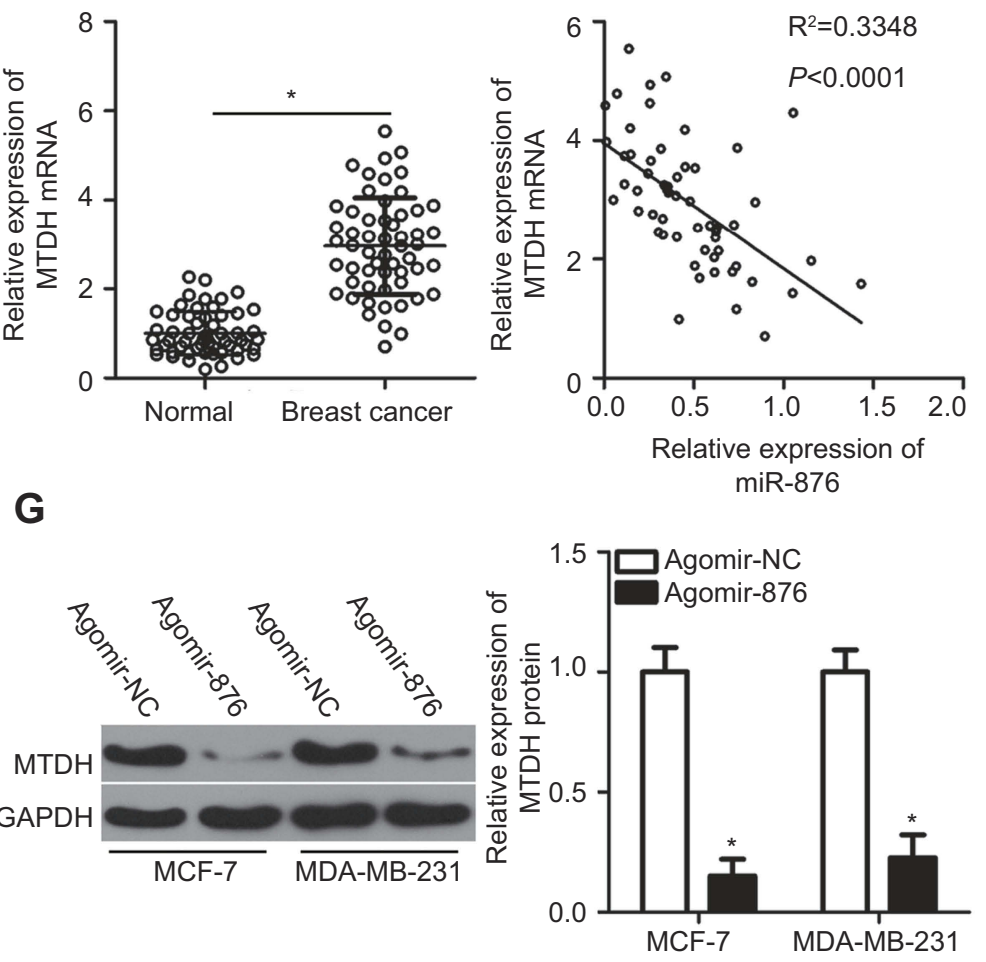

Figure 3 Identification of MTDH mRNA as a direct target of miR-876 in breast cancer. (A) The potential wild-type binding site of miR-876 in the $3^{\prime}$-UTR of MTDH mRNA, as predicted by three independent prediction algorithms. The mutant binding site is also shown. (B, C) MCF-7 and MDA-MB-23I cells were cotransfected with agomir-876 or agomir-NC and either MTDH-WT or MTDH-MUT. The luciferase reporter assay was carried out to determine luciferase activity. $* P<0.05$ as compared with agomir-NC. (D) RT-qPCR measurements of MTDH mRNA expression in 53 pairs of breast cancer samples and adjacent normal tissues. $* P<0.05$ as compared with normal tissues. (E) The expression correlation between miR-876 and MTDH mRNA in breast cancer tissue samples was evaluated by Spearman's correlation analysis. $R^{2}=0.3348, P<0.000$ I. $(F, G)$ The effects of miR-876 overexpression on MTDH mRNA and protein levels in MCF-7 and MDA-MB-23I cells were measured by RT-qPCR and Western blot analysis. *P<0.05 in comparison with agomir-NC. Abbreviations: agomir-876, miR-876 agomir; agomir-NC, negative control agomir; RT-qPCR, reverse transcription-quantitative polymerase chain reaction; MTDH, metadherin; WT, wild-type; MUT, mutant.

and MDA-MB-231 cells. By contrast, the decreased MTDH expression due to agomir-876 transfection was attenuated in the two cell lines cotransfected with pc-MTDH (Figure 4A, $P<0.05)$. Functionally, restoration of MTDH expression abrogated the decrease in cell proliferation (Figure 4B, $P<0.05$ ) and the increase in apoptosis (Figure $4 \mathrm{C}, P<0.05$ ) induced by miR876 overexpression in MCF-7 and MDA-MB-231 cells. Furthermore, the decrease in cell migration (Figure 4D, $P<0.05$ ) and invasion (Figure 4E, $P<0.05$ ) caused by agomir876 transfection was attenuated by restoration of MTDH expression. These results suggested that miR-876 exerts its anticancer activity in breast cancer by decreasing MTDH expression.

\section{LncRNA LINC00707 acts as a sponge for miR-876 in breast cancer cells}

It is well-known that lncRNA can act as a molecular sponge binding miRNAs and thereby titrating free miRNA. ${ }^{19}$ Thus, we hypothesized that miR-876 is sponged 
A

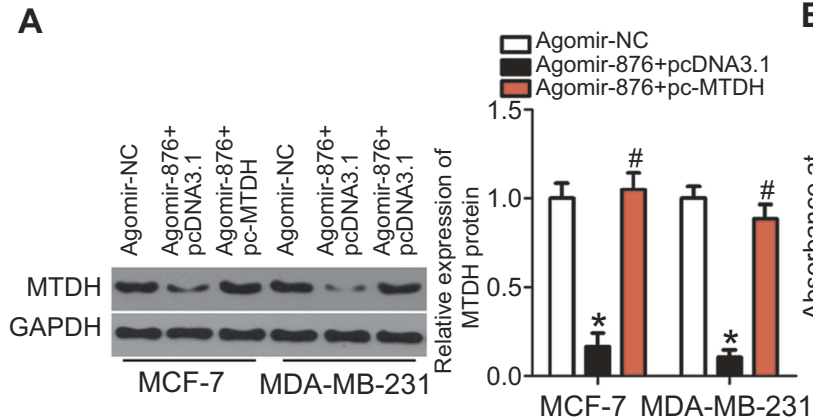

C

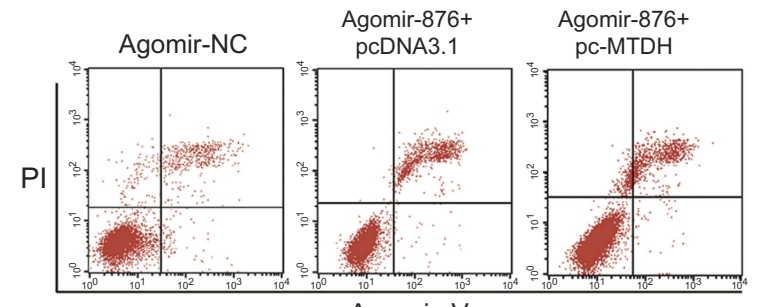

Annexin $\mathrm{V}$

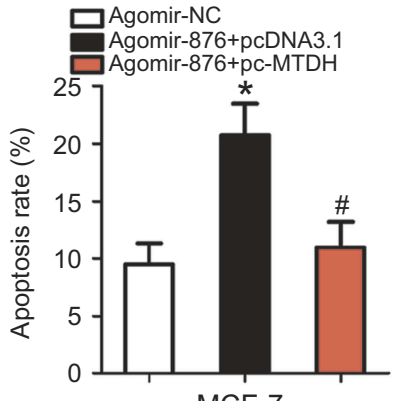

D

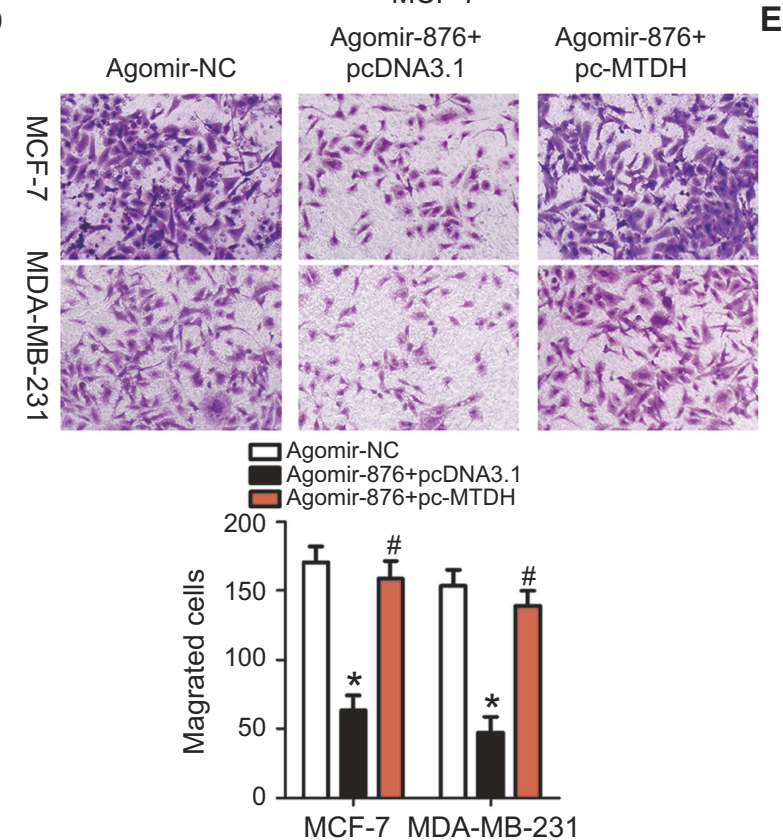

B

a Agomir-NC

$\rightarrow$ Agomir-876+pcDNA3.1

- Agomir-876+pc-MTDH
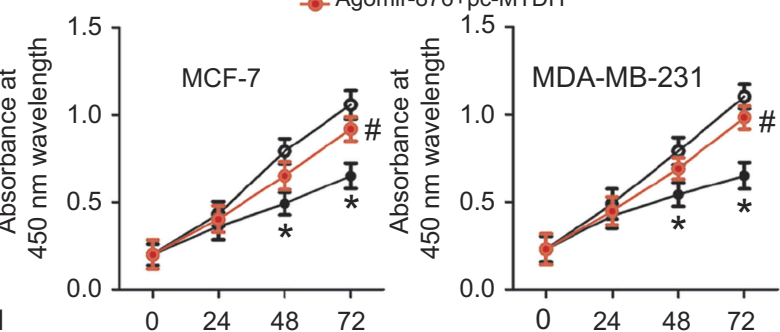

Time (hours after inoculation)
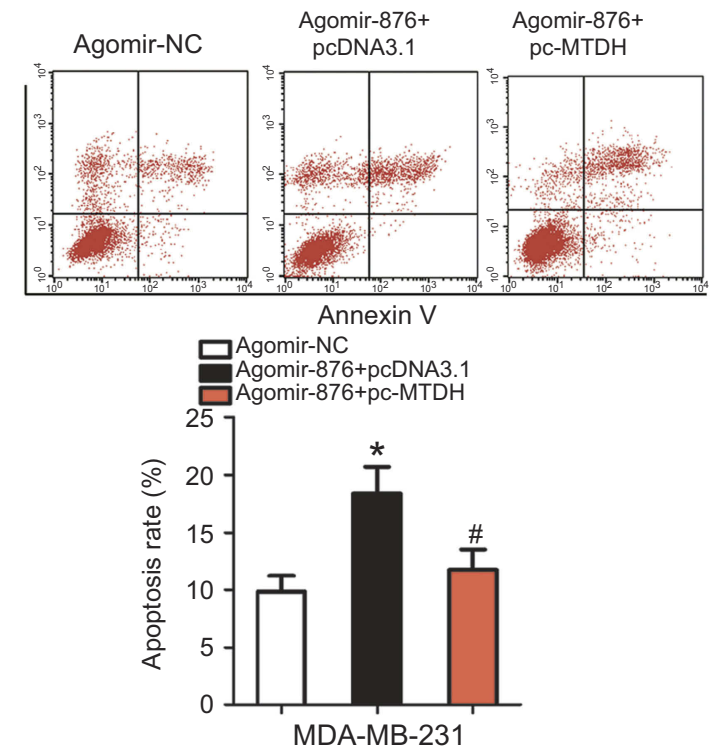

E Agomir-876+ ${ }^{-}$Agomir-876+
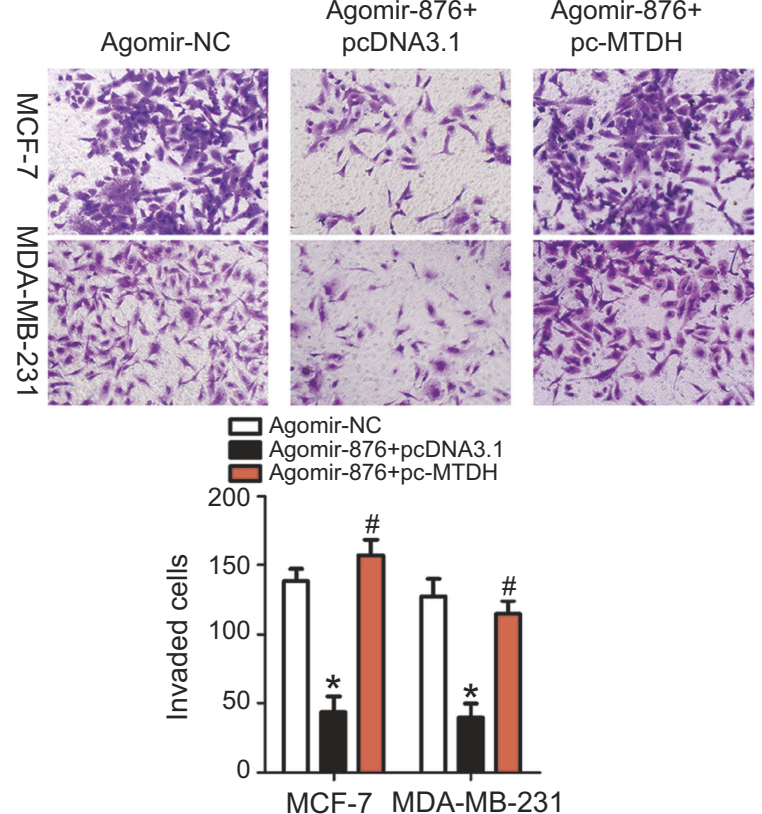

Figure 4 Restoring MTDH expression reverses the miR-876-mediated suppression of growth and metastasis of breast cancer cells. Agomir-876 along with pc-MTDH or pcDNA3.I was introduced into MCF-7 and MDA-MB-23I cells. The transfected cells were harvested after incubation at different time points and used in the following assays. (A) Total protein was extracted and used for the detection of MTDH protein expression. ${ }^{* P}<0.05$ as compared with agomir-NC. ${ }^{\#} P<0.05$ as compared with group agomir-876+pcDNA3.I. (B, C) Cell proliferation and invasion were measured by CCK-8 assays and flow cytometry. ${ }^{*} P<0.05$ relative to group agomir-NC. ${ }^{*} P<0.05$ as compared with agomir-876+ $P C D N A 3$. I. (D, E) The migration and invasion of MCF-7 and MDA-MB-23 I cells were quantified by Transwell migration and invasion assays. ${ }^{*} P<0.05$ as compared with agomir-NC. ${ }^{*} P<0.05$ in comparison with agomir-876+pcDNA3.I.

Abbreviations: agomir-876, miR-876 agomir; agomir-NC, negative control agomir; MTDH, metadherin. 
by some lncRNA, resulting in the restriction of breast cancer progression. To test this hypothesis, bioinformatics analysis was performed and two potential miR-876-binding sites in LINC00707 were identified (Figure 5A). The luciferase reporter assay was then carried out to confirm that miR-876 is a downstream target of LINC00707 in breast cancer cells. It was observed that miR-876 overexpression significantly suppressed the luciferase activity of LINC00707-WT (both 1 and 2) in MCF-7 and MDAMB-231 cells (Figure 5B; $P<0.05$ ). However, transfection with agomir-876 failed to affect the luciferase activity of LINC00707-MUT (both 1 and 2).

RT-qPCR analysis confirmed that LINC00707 expression was higher in breast cancer tissue samples than in adjacent normal tissue samples (Figure 5C, $P<0.05$ ). Importantly, LINC00707 expression negatively correlated with miR-876 expression in the same breast cancer tissue (Figure 5D; $\mathrm{R}^{2}=0.3697, P<0.0001$ ). In addition, si-LINC 00707-mediated silencing of LINC00707 expression (Figure 5E, $P<0.05$ ) significantly increased miR-876 expression (Figure 5F, $P<0.05$ ). Furthermore, Western blotting revealed that in MCF-7 and MDA-MB-231 cells, transfection with si-LINC00707 significantly downregulated MTDH protein (Figure $5 \mathrm{G}, P<0.05$ ). Taken together, these results confirmed that LINC00707 acts as a molecular sponge for miR-876 to regulate $\mathrm{MTDH}$ expression in breast cancer.

\section{LINC00707 knockdown inhibits the proliferation, migration and invasiveness and induces the apoptosis of breast cancer cells}

To explore the specific roles of LINC00707 in breast cancer, si-LINC00707 was transfected to silence LINC00707 expression in MCF-7 and MDA-MB-231 cells. si-NC acted as the control for si-LINC00707. Predictably, the LINC00707 knockdown significantly decreased the proliferation of MCF-7 and MDA-MB-231 cells (Figure 6A, $P<0.05$ ). In addition, LINC00707depleted MCF-7 and MDA-MB-231 cells showed significantly more apoptosis (Figure 6B, $P<0.05$ ). Furthermore, Transwell migration and invasion assays indicated that the number of cells that migrated (Figure 6C, $P<0.05$ ) and invaded (Figure 6D, $P<0.05$ ) was lower in the si-LINC 00707 group than in the si-NC group. These findings confirmed that LINC00707 has tumor-promoting effects on the growth and metastasis of breast cancer in vitro.

\section{miR-876 is responsible for the tumor-promoting functions of LINC00707 in breast cancer cells}

To determine whether the effects of LINC00707 silencing in breast cancer cells were mediated by miR-876, MCF-7 and MDA-MB-231 cells were cotransfected with si-LINC00707 and antagomir-876 or antagomir-NC; then cell proliferation, apoptosis, migration and invasion were examined by CCK-8, flow cytometric and Transwell migration and invasion assays, respectively. The increased miR-876 expression (Figure 7A, $P<0.05$ ) and decreased MTDH protein expression (Figure $7 \mathrm{~B}, P<0.05$ ) in the LINC00707 knockdown MCF-7 and MDA-MB-231 cells were reversed by cotransfection with antagomir-876, as revealed by RT-PCR and Western blotting. Functional experiments revealed that the loss of LINC00707 significantly inhibited cell proliferation (Figure 7C, $P<0.05$ ), induced apoptosis (Figure 7D, $P<0.05$ ) and decreased migration (Figure $7 \mathrm{E}, P<0.05$ ) and invasiveness (Figure 7F, $P<0.05$ ) in MCF-7 and MDA-MB-231 cells. However, cotransfection with antagomir-876 abrogated these effects of LINC00707 silencing. In summary, these results suggested that LINC00707 is implicated in the regulation of breast cancer progression by regulating miR-876 activity.

\section{MiR-876 upregulation decreases tumor growth of breast cancer cells in vivo}

Tumor xenograft experiments were conducted to examine the function of miR-876 overexpression in breast cancer tumor growth in vivo. The volumes (Figure $8 \mathrm{~A}$ and $\mathrm{B}$, $P<0.05$ ) and weights (Figure $8 \mathrm{C}, P<0.05$ ) of tumor xenografts were both smaller in the agomir-876 group than in the agomir-NC group. In addition, RT-qPCR analysis showed that miR-876 expression in tumor xenografts from the agomir-876 group was higher than that in the agomir-NC group (Figure 8D, $P<0.05$ ). Then, we measured the MTDH protein levels in the formed tumor xenografts. MTDH protein expression was also downregulated in vivo after miR-876 upregulation (Figure $8 \mathrm{E}, P<0.05$ ). These results suggested that miR-876 exerts an inhibitory action on the tumor growth of breast cancer cells in vivo.

\section{Discussion}

In recent years, aberrant miRNA expression has been identified in breast cancer, which has important roles in the progression of malignancy. ${ }^{28}$ miRNAs can act as tumor suppressors or oncogenic molecules depending on the biological functions of their targets. ${ }^{29}$ Therefore, exploring 
A

$\begin{array}{lc} & \\ \text { LINC00707 wild-type } & 5^{\prime} \\ \text { hsa-miR-876 } & 3^{\prime} \\ \text { LINC00707 mutant } & 5^{\prime} \\ & \\ \text { LINC00707 wild-type } & 5^{\prime} \\ \text { hsa-miR-876 } & 3^{\prime} \\ \text { LINC00707 mutant } & 5^{\prime}\end{array}$

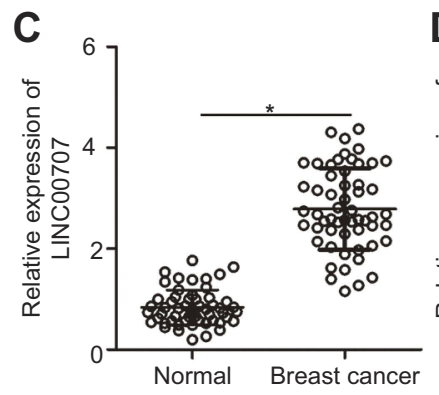

Site 1

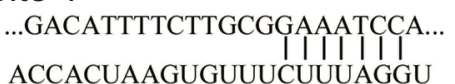

...GACATTTTCTTGCGCUUUAGGA...

\section{Site 2}

...CGGAAATCCA-AAAGAAATCCC.. ACCACUAAGUGUUUCUUUAGGU

...CGGAAATCCAAAACUUUAGGC..
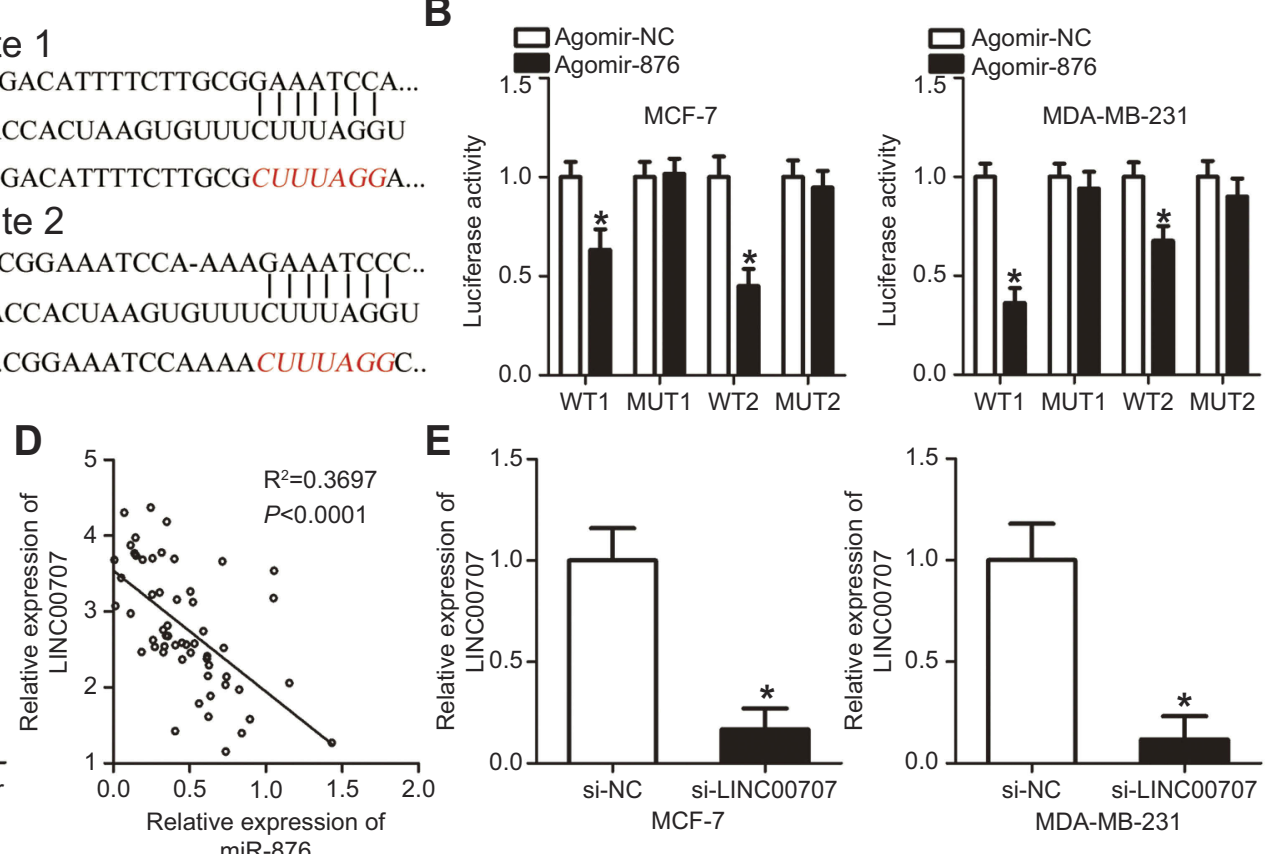

\section{F}

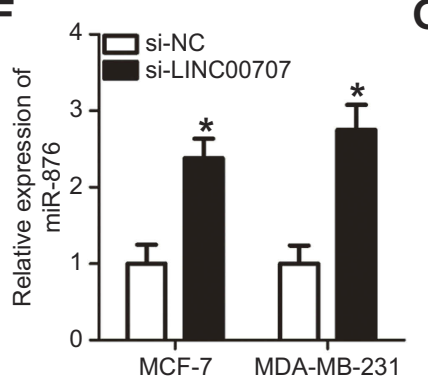

G

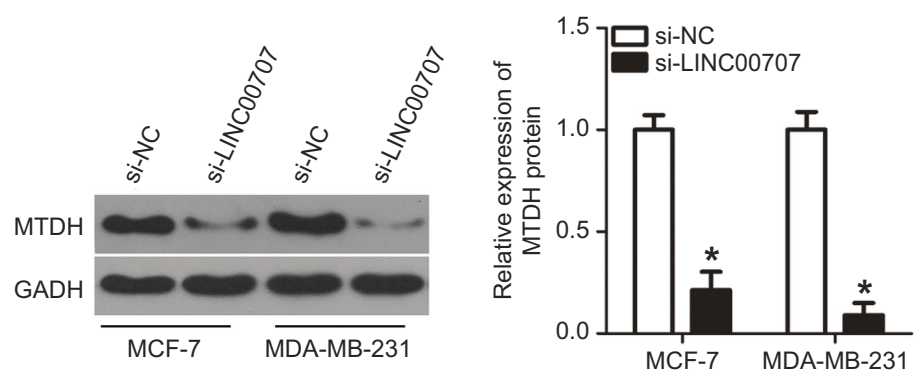

Figure 5 LINC00707 functions as a ceRNA for miR-876 in breast cancer cells. (A) Schematic illustration of the wild-type and mutant miR-876-binding sites in LINC00707. (B) Luciferase activity in MCF-7 and MDA-MB-23I cells after cotransfection with agomir-876 or agomir-NC and LINC00707-WT or LINC00707-MUT was measured by luciferase reporter assays. $* P<0.05$ as compared with agomir-NC. (C) LINC00707 expression was determined in 53 pairs of breast cancer samples and adjacent normal tissues. ${ }^{*} P<0.05$ as compared with normal tissues. (D) Spearman's correlation analysis uncovered a negative correlation between miR-876 and LINC00707 in breast cancer tissue samples. $R^{2}=0.3697, P<0.000$ I. (E) si-LINC00707 or si-NC was transfected into MCF-7 and MDA-MB-23I cells. After cultivation for 48 hrs, transfection efficiency was evaluated by RT-qPCR. $* P<0.05$ relative to group si-NC. (F) The expression of miR-876 in the LINC00707 knockdown MCF-7 and MDA-MB-23I cells was detected via RTqPCR. $* P<0.05$ as compared with si-NC. (G) MTDH protein expression in the LINC00707 knockdown MCF-7 and MDA-MB-23I cells was evaluated via Western blotting. $* P<0.05$ as compared with si-NC.

Abbreviations: MTDH, metadherin; si-LINC00707, small interfering RNA (siRNA) targeting LINC00707; si-NC, negative control siRNA; ceRNA, competing endogenous RNA; WT, wild-type; MUT, mutant.

the functions of cancer-specific miRNAs in breast cancer and their mechanisms of action is needed to identify effective targets for anticancer therapy. In this study, for the first time, we measured the expression of miR-876 in breast cancer and evaluated its clinical value. In addition, the detailed roles of miR-876 in the progression of breast cancer were explored using a series of experiments. Notably, another aim of this study was to determine the mechanisms underlying the activity of miR-876 in breast cancer progression, which might be helpful for identifying promising targets for novel treatments.

miR-876 is downregulated in osteosarcoma, and its downregulation positively correlates with an advanced clinical stage and poor differentiation status. ${ }^{23}$ Patients with osteosarcomas that have low miR-876 expression exhibit worse overall survival than patients with high miR-876 expression. ${ }^{23}$ miR-876 is also expressed at low levels in hepatocellular carcinoma, ${ }^{24}$ lung cancer, ${ }^{25}$ and head and neck squamous cell carcinoma. ${ }^{26}$ Nevertheless, to date, few studies have focused on the expression profile of miR-876 in breast cancer. Here, we found that miR-876 expression is low in breast cancer tissues and cell lines. Low miR-876 expression correlated with lymphatic invasion metastasis, TNM stage and differentiation grade. More importantly, patients with breast cancer containing low levels of miR-876 showed lower 
A

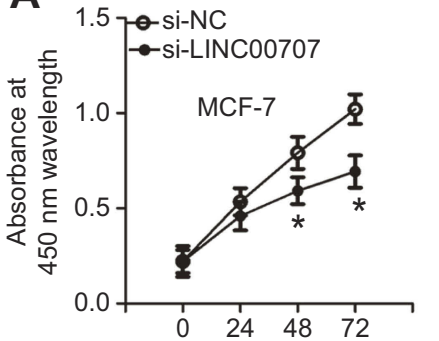

Time (hours after inoculation)

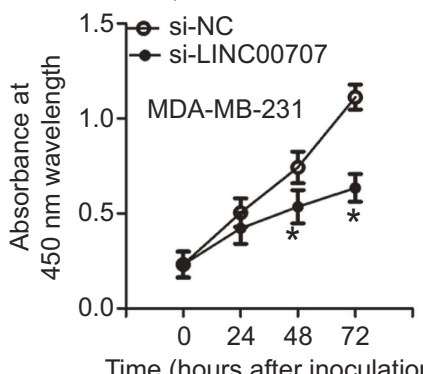

C
B
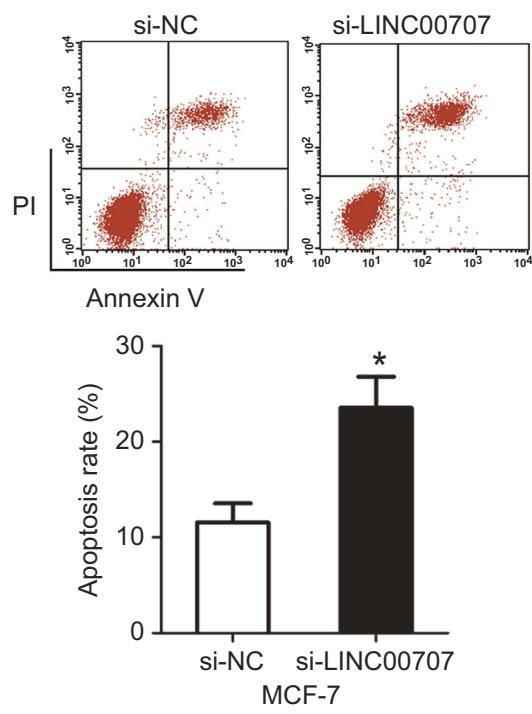

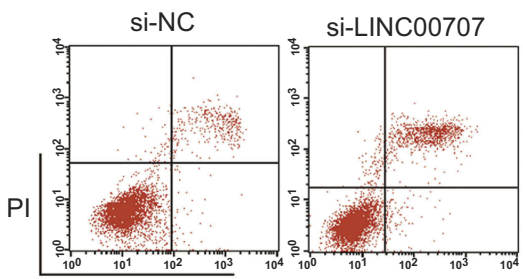

Annexin $\mathrm{V}$

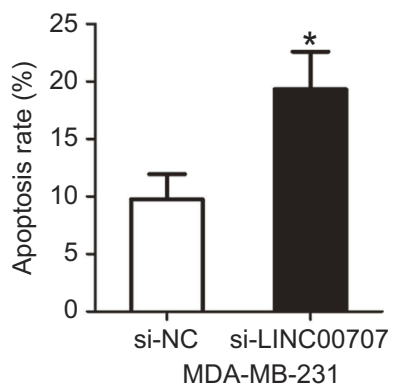

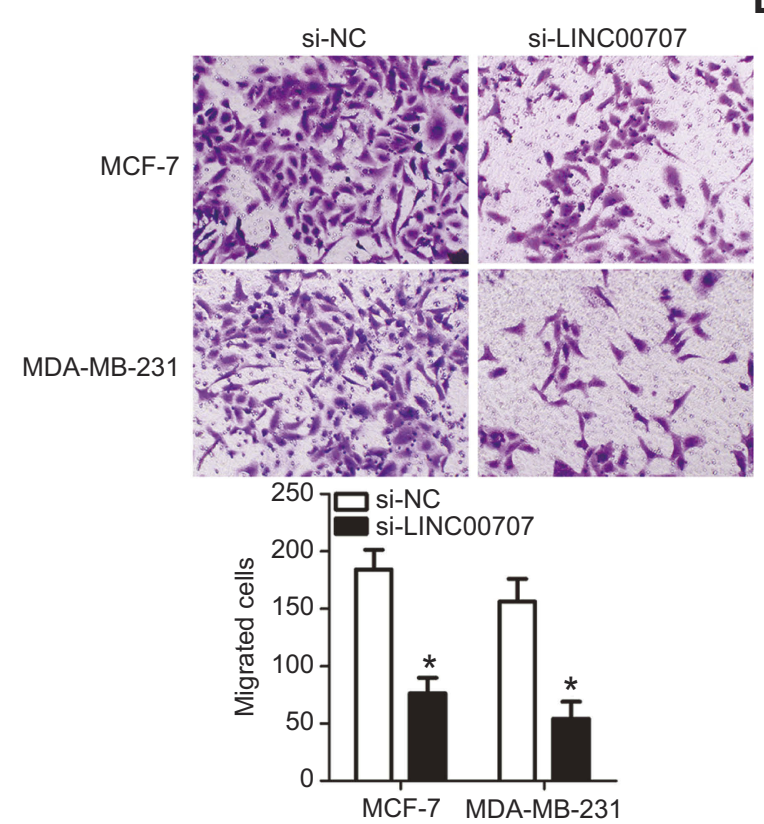

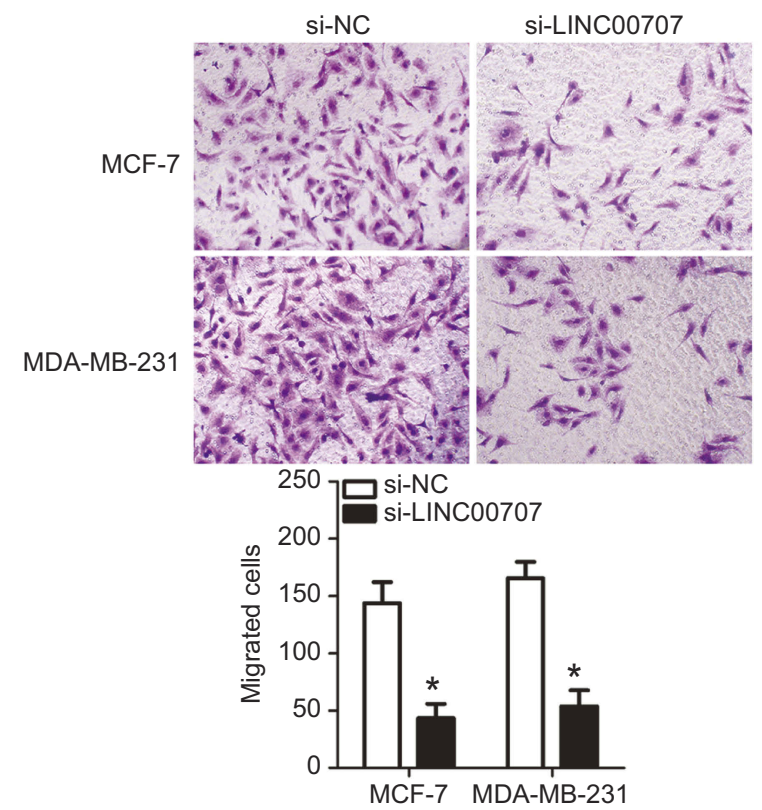

Figure 6 Silencing of LINC00707 expression inhibits the growth and metastasis of breast cancer cells in vitro. MCF-7 and MDA-MB-23I cells were transfected with si-LINC 00707 or si-NC and used in the following functional experiments. (A) The proliferation of MCF-7 and MDA-MB-23I cells after LINC00707 depletion was determined by the CCK-8 assay. ${ }^{* P}<0.05$ in comparison with si-NC. (B) The apoptosis rate of MCF-7 and MDA-MB-23I cells as measured by the annexin V-FITC apoptosis detection kit. $* P<0.05$ as compared with si-NC. (C, D) The impact of LINC00707 silencing on the migration and invasiveness of MCF-7 and MDA-MB-23I cells as measured by Transwell migration and invasion assays. $* P<0.05$ as compared with si-NC.

Abbreviations: MTDH, metadherin; si-LINC00707, small interfering RNA (siRNA) targeting LINC00707; si-NC, negative control siRNA; CCK-8, Cell Counting Kit-8 assay.

overall survival than patients with high levels of miR-876 expression. These observations suggest that miR-876 might be an effective diagnostic and prognostic biomarker for patients with breast cancer.

miR-876 has tumor-suppressive roles in the carcinogenesis and cancer progression. For instance, miR-876 upregulation restricts osteosarcomas cell proliferation, migration and invasion in vitro and hinders tumor growth in vivo. ${ }^{23}$ Ectopic miR-876 expression suppresses cell metastasis in head and neck squamous cell carcinoma both in vitro and in vivo. ${ }^{26}$ Furthermore, miR- 876 has been validated as a tumor-suppressing miRNA in hepatocellular carcinoma ${ }^{24}$ and lung cancer. ${ }^{25}$ However, whether miR-876 is involved in breast cancer progression has remained unknown until now. 

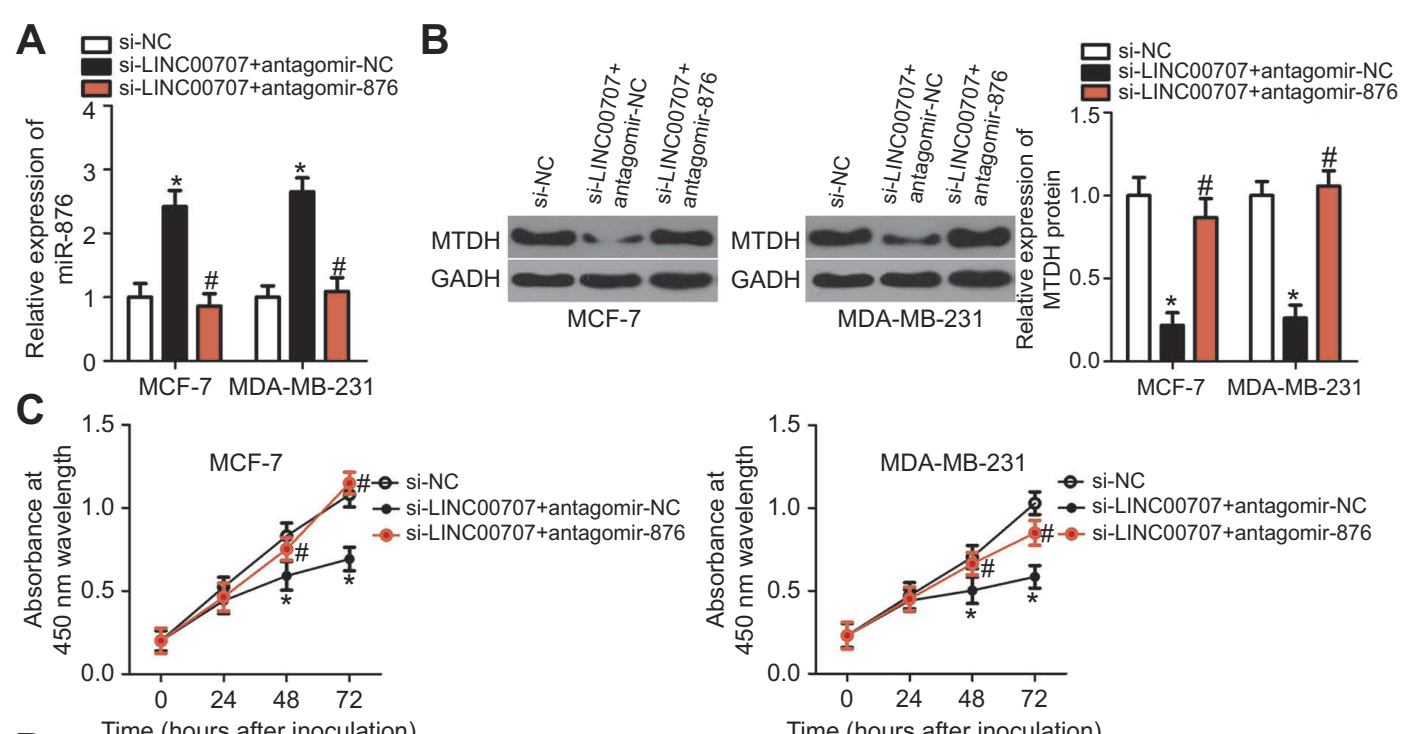

D Time (hours after inoculation)

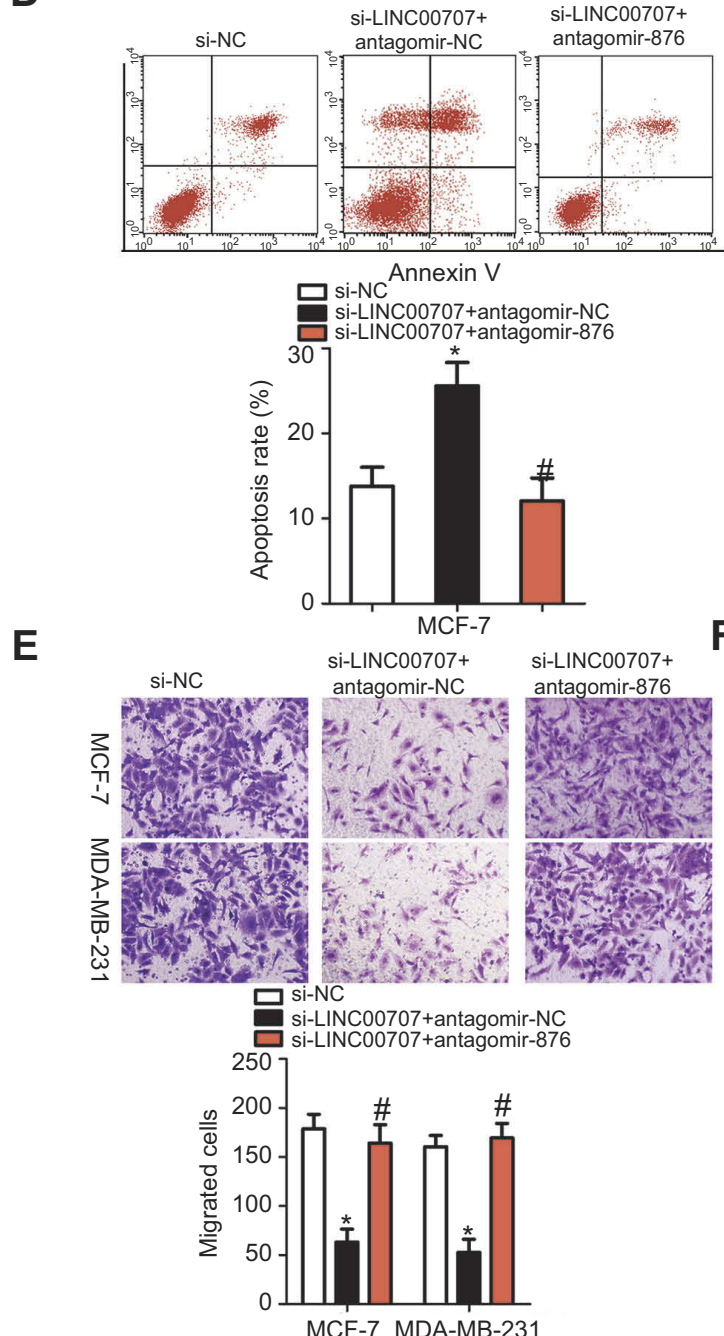

Time (hours after inoculation)
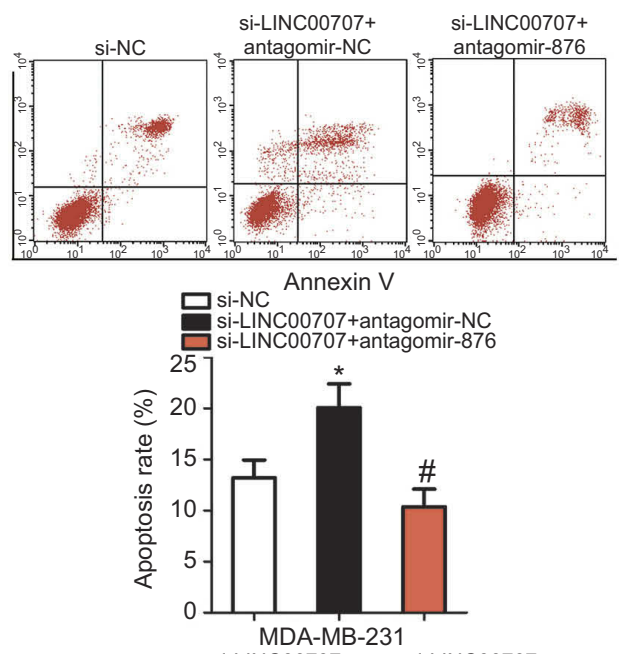

$\mathbf{F}$
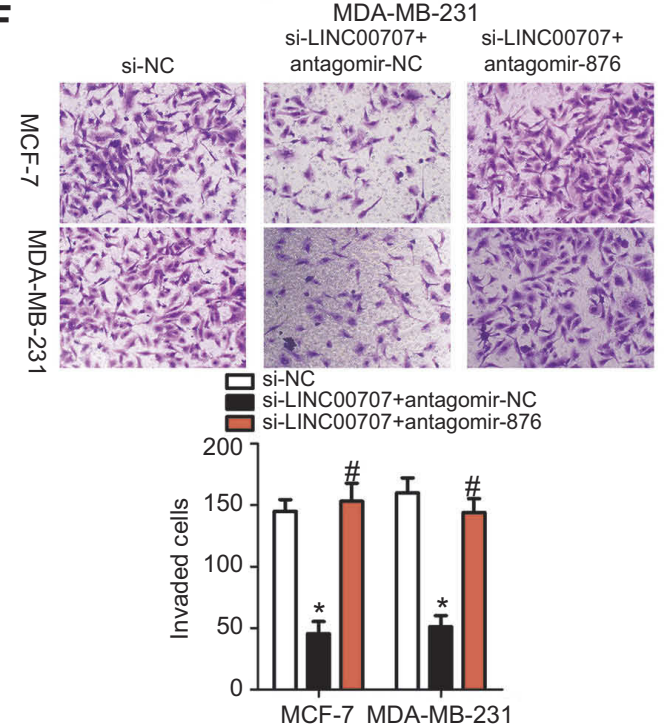

Figure 7 miR-876 knockdown neutralizes the impact of si-LINC00707 on breast cancer cells. (A, B) MCF-7 and MDA-MB-23I cells were cotransfected with si-LINC00707 in the presence of antagomir-876 or antagomir-NC. RT-qPCR and Western blot analysis were conducted to determine the levels of miR-876 and MTDH protein expression. $* P<0.05$ as compared with si-NC. ${ }^{\#}$ P $<0.05$ relative to group si-LINC00707+antagomir-NC. (C-F) Cell proliferation, apoptosis, migration and invasiveness of MCF-7 and MDA-MB-23I cells treated as described above were determined by CCK-8, flow cytometric and Transwell migration and invasion assays, respectively. ${ }^{*}<<0.05$ as compared with si-NC. ${ }^{\#} P<0.05$ as compared with si-LINC00707+antagomir-NC.

Abbreviations: MTDH, metadherin; si-LINC00707, small interfering RNA (siRNA) targeting LINC00707; si-NC, negative control siRNA; CCK-8, Cell Counting Kit-8 assay; agomir876, miR-876 agomir; agomir-NC, negative control agomir. 
A

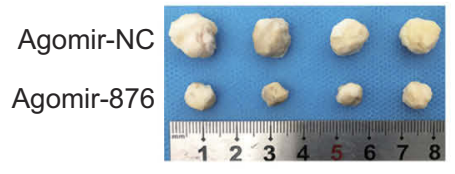

D

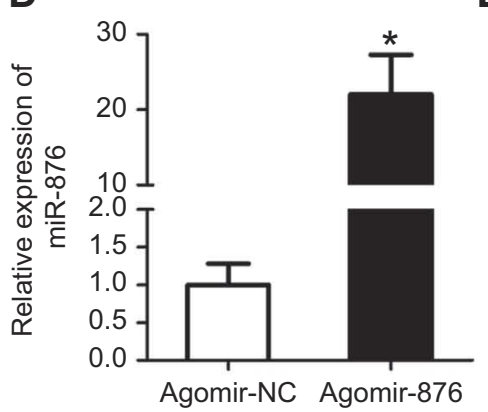

B

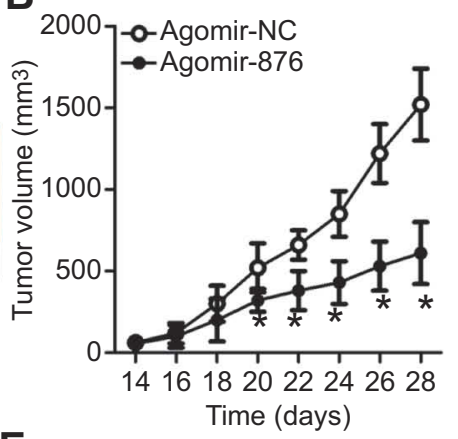

E

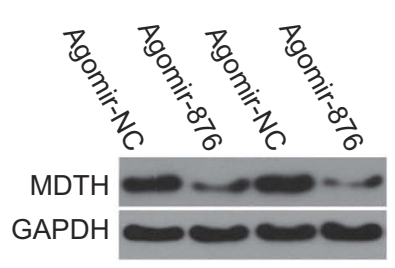

C

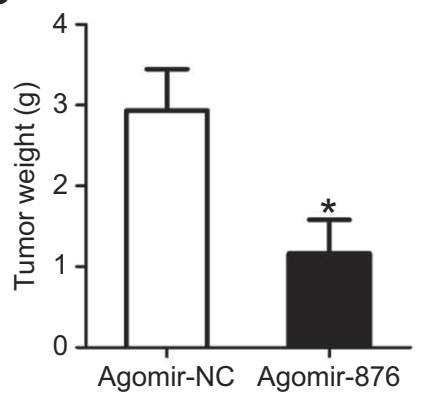

Figure 8 miR-876 upregulation inhibits breast cancer cell-derived tumor growth in vivo. (A) Photographs of tumor xenografts derived from agomir-876- and agomir-NCtransfected MCF-7 cells. (B, C) Comparison of the tumor volume and weight between the agomir-876 and agomir-NC groups. $* P<0.05$ in comparison with agomir-NC. (D) The expression levels of miR-876 in the tumor xenografts were examined by RT-qPCR. $* P<0.05$ as compared with agomir-NC. (E) Western blot analysis confirmed the difference in MTDH protein expression between the agomir-876 and agomir-NC groups. $* P<0.05$ relative to group agomir-NC.

Abbreviations: agomir-876, miR-876 agomir; agomir-NC, negative control agomir; miR-876, microRNA-876; RT-qPCR, reverse-transcription quantitative polymerase chain reaction; MTDH, metadherin.

In this study, restoring miR-876 expression decreased the proliferation, migration and invasion of breast cancer cells in vitro and promoted cell apoptosis. In addition, miR-876 overexpression dramatically delayed the growth of breast cancer-derived tumors in vivo. Accordingly, miR-876 has potential as an effective target for breast cancer therapy.

Multiple genes, including c-Met in osteosarcoma ${ }^{23}$ BCL6 corepressor-like $1,{ }^{24}$ DNA (cytosine-5)-methyltransferase $3 \mathrm{~A}^{30}$ in hepatocellular carcinoma and bone morphogenetic protein 4 in lung cancer, ${ }^{25}$ have been identified as direct target genes of miR-876. Currently, the lncRNA-miRNA-mRNA axis is the most typical regulatory mechanism for lncRNA. Here, $M T D H$ mRNA was identified as a direct target of miR876 in breast cancer cells, with LINC00707 acting as a ceRNA for miR-876 to regulate MTDH expression. MTDH is upregulated in breast cancer and its overexpression is related to nuclear grade, negative estrogen receptor and progesterone receptor expression, disease-free survival, distant-metastasisfree survival and overall survival. ${ }^{31}$ Multivariate analysis identified MTDH as an independent biomarker of poor disease-free survival and distant metastasis-free survival rates. ${ }^{31} \mathrm{MTDH}$ functions in many biological processes closely related to breast cancer formation and progression. ${ }^{32-35}$ Therefore, MTDH silencing due to miR-876 overexpression might be an attractive therapeutic technique in patients with breast cancer.

LINC00707 expression is high in lung adenocarcinomas, and its upregulation is associated with advanced TNM stage, larger tumor size, lymphatic metastasis and poor prognosis. ${ }^{36}$ LINC00707 is upregulated in gastric cancer, and this increase correlates with tumor stage, tumor size, lymph node metastasis and worsened prognosis. ${ }^{37}$ Several studies have shown that LINC00707 is a key regulatory lncRNA in lung adenocarcinomas, ${ }^{36}$ gastric cancer $^{37}$ and hepatocellular carcinoma. ${ }^{38,39}$ These tumor-promoting actions work through multiple mechanisms, including downregulation of $\mathrm{Cdc} 42$ in lung adenocarcinomas, ${ }^{36}$ interaction with mRNA-stabilizing protein $\mathrm{HuR}$ in gastric cancer ${ }^{37}$ and activation of the ERK-JNK-AKT pathway ${ }^{38}$ and sponging miR-206 ${ }^{39}$ in hepatocellular carcinoma. Herein, we demonstrated the upregulation of LINC00707 in breast cancer and evaluated its effect. LINC00707 promoted the oncogenicity of breast cancer cells through sponging miR-876 and subsequently regulating MTDH expression. Hence, LINC00707, miR876 and MTDH may be interrelated and, thus, regulate the aggressiveness of breast cancer. 


\section{Conclusion}

We proved that miR-876 is downregulated in breast cancer tissues and cell lines, and that its decreased expression is associated with poor prognosis among patients with breast cancer. This study also uncovered the role of the LINC00707-miR-876-MTDH pathway in the malignant progression of breast cancer and provided novel targets for the management of breast cancer. However, we did not explore the effect of miR-873 on the metastasis of breast cancer in vivo. It is a limitation of our study, and we will resolve it in our future investigation.

\section{Disclosure}

The authors report no conflicts of interest in this work.

\section{References}

1. Hamann U, Ankel C. [Breast cancer: diagnostics and therapy - the most important facts for internists]. Deutsche Medizinische Wochenschrift. 2018;143(4):267-278. doi:10.1055/s-0043-104456

2. Varghese F, Wong J. Breast cancer in the elderly. Surg Clin North Am. 2018;98(4):819-833. doi:10.1016/j.suc.2018.04.002

3. Kluthcovsky AC, Faria TN, Carneiro FH, Strona R. Female breast cancer mortality in Brazil and its regions. Rev Assoc Med Bras. 2014;60 (4):387-393.

4. Lin X, Chen W, Wei F, Ying M, Wei W, Xie X. Night-shift work increases morbidity of breast cancer and all-cause mortality: a meta-analysis of 16 prospective cohort studies. Sleep Med. 2015;16 (11):1381-1387. doi:10.1016/j.sleep.2015.02.543

5. Chaffer CL, Weinberg RA. A perspective on cancer cell metastasis. Science. 2011;331(6024):1559-1564. doi:10.1126/science.1203543

6. Eckhardt BL, Francis PA, Parker BS, Anderson RL. Strategies for the discovery and development of therapies for metastatic breast cancer. Nat Rev Drug Discov. 2012;11(6):479-497. doi:10.1038/nrd2372

7. Musgrove EA, Sutherland RL. Biological determinants of endocrine resistance in breast cancer. Nat Rev Cancer. 2009;9(9):631-643. doi: $10.1038 / \mathrm{nrc} 2713$

8. Raval GN, Bharadwaj S, Levine EA, et al. Loss of expression of tropomyosin-1, a novel class II tumor suppressor that induces anoikis, in primary breast tumors. Oncogene. 2003;22(40):6194-6203. doi:10.1038/sj.onc. 1206719

9. Volinsky N, McCarthy CJ, von Kriegsheim A, et al. Signalling mechanisms regulating phenotypic changes in breast cancer cells. Biosci Rep. 2015;35(2). doi:10.1042/BSR20150111.

10. Sauter ER. Breast cancer prevention: current approaches and future directions. Eur J Breast Health. 2018;14(2):64-71. doi:10.5152/ ejbh.2018.3978

11. Esquela-Kerscher A, Slack FJ. Oncomirs - microRNAs with a role in cancer. Nat Rev Cancer. 2006;6(4):259-269. doi:10.1038/nrc1840

12. Sempere LF, Keto J, Fabbri M. Exosomal microRNAs in breast cancer towards diagnostic and therapeutic applications. Cancers. 2017;9(7):71. doi:10.3390/cancers9070071

13. Delsin LEA, Salomao KB, Pezuk JA, Brassesco MS. Expression profiles and prognostic value of miRNAs in retinoblastoma. $J$ Cancer Res Clin Oncol. 2019;145(1):1-10. doi:10.1007/s00432-018-2773-7

14. Link A, Kupcinskas J. MicroRNAs as non-invasive diagnostic biomarkers for gastric cancer: current insights and future perspectives. World J Gastroenterol. 2018;24(30):3313-3329. doi:10.3748/wjg. v24.i30.3313
15. To KK, Tong CW, Wu M, Cho WC. MicroRNAs in the prognosis and therapy of colorectal cancer: from bench to bedside. World J Gastroenterol. 2018;24(27):2949-2973. doi:10.3748/wjg.v24.i27.2949

16. Kong P, Chen L, Yu M, et al. miR-3178 inhibits cell proliferation and metastasis by targeting Notch1 in triple-negative breast cancer. Cell Death Dis. 2018;9(11):1059. doi:10.1038/s41419-018-1091-y

17. Zhang Y, Fang J, Zhao H, Yu Y, Cao X, Zhang B. Downregulation of microRNA-1469 promotes the development of breast cancer via targeting HOXA1 and activating PTEN/PI3K/AKT and Wnt/beta-catenin pathways. J Cell Biochem. 2019;120(4):5097-5107. doi:10.1002/ jcb. 27786

18. Li J, Lu M, Jin J, Lu X, Xu T, Jin S. miR-449a suppresses tamoxifen resistance in human breast cancer cells by targeting ADAM22. Cell Physiol Biochem. 2018;50(1):136-149. doi:10.1159/000493964

19. Quan M, Chen J, Zhang D. Exploring the secrets of long noncoding RNAs. Int J Mol Sci. 2015;16(3):5467-5496. doi:10.3390/ ijms 16035467

20. Jiao D, Li Z, Zhu M, Wang Y, Wu G, Han X. LncRNA MALAT1 promotes tumor growth and metastasis by targeting miR-124/foxq1 in bladder transitional cell carcinoma (BTCC). Am J Cancer Res. 2018;8(4):748-760.

21. Ma J, Fan Y, Feng T, et al. HOTAIR regulates HK2 expression by binding endogenous miR-125 and miR-143 in oesophageal squamous cell carcinoma progression. Oncotarget. 2017;8(49):86410-86422. doi:10.18632/oncotarget.21195

22. Li Y, Han X, Li Q, Wang C, Lou Z, Wang X. Long noncoding RNA HOXD-AS1 induces epithelial-mesenchymal transition in breast cancer by acting as a competing endogenous RNA of miR-421. J Cell Biochem. 2019;120(6):10633-10642.

23. Xie W, Xiao J, Wang T, Zhang D, Li Z. MicroRNA-876-5p inhibits cell proliferation, migration and invasion by targeting c-Met in osteosarcoma. J Cell Mol Med. 2019;23:3293-3301. doi:10.1111/ jcmm.2019.23.issue-5

24. Xu Q, Zhu Q, Zhou Z, et al. MicroRNA-876-5p inhibits epithelial-mesenchymal transition and metastasis of hepatocellular carcinoma by targeting BCL6 corepressor like 1. Biomed Pharmacother. 2018;103:645-652. doi:10.1016/j.biopha.2018 .04 .037

25. Bao L, Lv L, Feng J, et al. MiR-876-5p suppresses epithelialmesenchymal transition of lung cancer by directly down-regulating bone morphogenetic protein 4. J Biosci. 2017;42(4):671-681.

26. Dong Y, Zheng Y, Wang C, et al. MiR-876-5p modulates head and neck squamous cell carcinoma metastasis and invasion by targeting vimentin. Cancer Cell Int. 2018;18:121. doi:10.1186/s12935-018-0619-7

27. Livak KJ, Schmittgen TD. Analysis of relative gene expression data using real-time quantitative PCR and the 2(-Delta delta C(T)) method. Methods. 2001;25(4):402-408. doi:10.1006/meth.2001.1262

28. Khordadmehr M, Shahbazi R, Ezzati H, Jigari-Asl F, Sadreddini S, Baradaran B. Key microRNAs in the biology of breast cancer; emerging evidence in the last decade. J Cell Physiol. 2019;234 (6):8316-8326. doi:10.1002/jcp. 27716

29. Mandujano-Tinoco EA, Garcia-Venzor A, Melendez-Zajgla J, Maldonado V. New emerging roles of microRNAs in breast cancer. Breast Cancer Res Treat. 2018;171(2):247-259. doi:10.1007/s10549018-4850-7

30. Wang Y, Xie Y, Li X, et al. MiR-876-5p acts as an inhibitor in hepatocellular carcinoma progression by targeting DNMT3A. Pathol Res Pract. 2018. doi:10.1016/j.prp.2018.04.012

31. Tokunaga E, Nakashima Y, Yamashita N, et al. Overexpression of metadherin/MTDH is associated with an aggressive phenotype and a poor prognosis in invasive breast cancer. Breast Cancer. 2014;21 (3):341-349. doi:10.1007/s12282-012-0398-2

32. Li J, Yang L, Song L, et al. Astrocyte elevated gene-1 is a proliferation promoter in breast cancer via suppressing transcriptional factor FOXO1. Oncogene. 2009;28(36):3188-3196. doi:10.1038/onc.2009.171 
33. Qian BJ, Yan F, Li N, et al. MTDH/AEG-1-based DNA vaccine suppresses lung metastasis and enhances chemosensitivity to doxorubicin in breast cancer. Cancer Immunol Immunother. 2011;60 (6):883-893. doi:10.1007/s00262-011-0997-3

34. Li X, Kong X, Huo Q, et al. Metadherin enhances the invasiveness of breast cancer cells by inducing epithelial to mesenchymal transition. Cancer Sci. 2011;102(6):1151-1157. doi:10.1111/j.1349-7006. 2011.01919.x

35. Wang C, Yang Q. Astrocyte elevated gene-1 and breast cancer (Review). Oncol Lett. 2011;2(3):399-405. doi:10.3892/ ol.2011.268

36. Ma T, Ma H, Zou Z, et al. The long intergenic noncoding RNA 00707 promotes lung adenocarcinoma cell proliferation and migration by regulating Cdc42. Cell Physiol Biochem. 2018;45(4):1566-1580. doi: $10.1159 / 000487693$
37. Xie M, Ma T, Xue J, et al. The long intergenic non-protein coding RNA 707 promotes proliferation and metastasis of gastric cancer by interacting with mRNA stabilizing protein HuR. Cancer Lett. 2019;443:67-79. doi:10.1016/j.canlet.2018.11.032

38. Wang J, Luo Z, Yao T, Li W, Pu J. LINC00707 promotes hepatocellular carcinoma progression through activating ERK/JNK/AKT pathway signaling pathway. J Cell Physiol. 2019;234(5):6908-6916. doi:10.1002/ jcp. 27449

39. Tu J, Zhao Z, Xu M, et al. LINC00707 contributes to hepatocellular carcinoma progression via sponging miR-206 to increase CDK14. J Cell Physiol. 2018;234(7):10615-10624.

\section{Publish your work in this journal}

Cancer Management and Research is an international, peer-reviewed open access journal focusing on cancer research and the optimal use of preventative and integrated treatment interventions to achieve improved outcomes, enhanced survival and quality of life for the cancer patient.
The manuscript management system is completely online and includes a very quick and fair peer-review system, which is all easy to use. Visit http://www.dovepress.com/testimonials.php to read real quotes from published authors. 